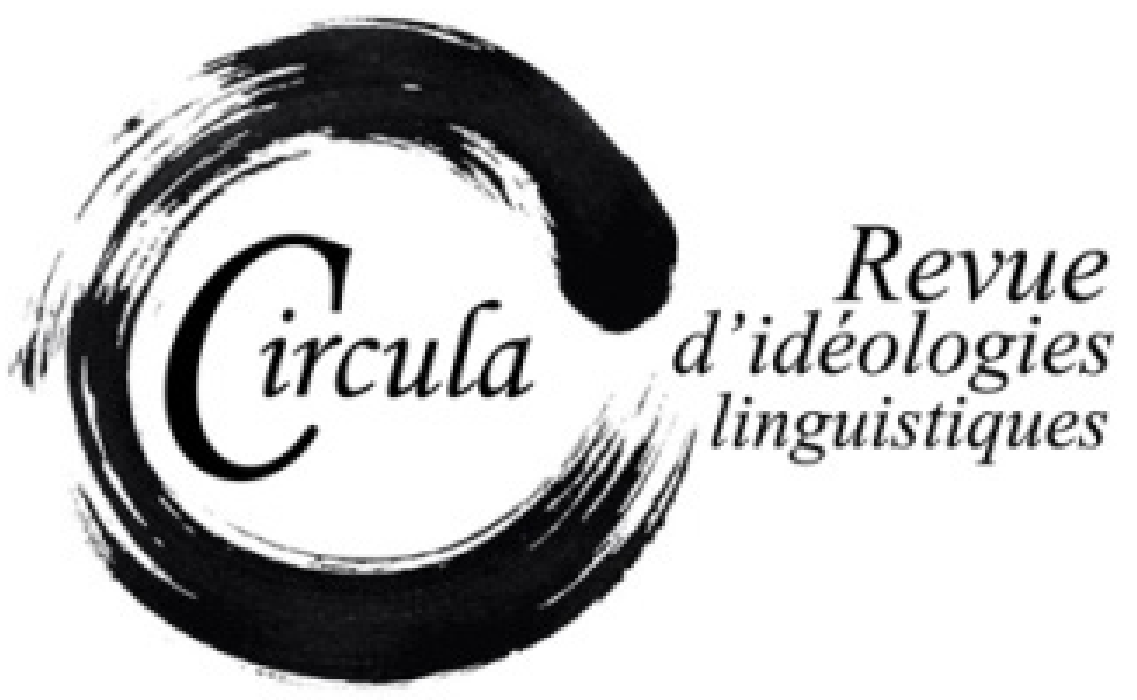

Titre: HiÉRARCHIES IMAgINÉES DES LOCUTEURS ET DES LANGUES-CULTURES AU MEXIQUE Auteur: Claudia Torres Castillo, Universidad Autónoma del Estado de México

ReVue: Circula, NUMÉRO 12 : LA VULNÉRABILITÉ LINGUISTIQUE

DiRECTRICE: Claudia TORRES CASTILLO

PAGES: $41-64$

ISSN: 2369-6761

URI: HTTP://HDL.HANDLE.NET/11143/18442

DOI: HTTPS://DOI.ORG/10.17118/11143/18442 


\title{
Hiérarchies imaginées des locuteurs et des langues-cultures au Mexique
}

\author{
Claudia Torres Castillo, Universidad Autónoma del Estado de México
}

claudiatorresc@yahoo.com.mx

\begin{abstract}
Résumé : L'image ou les images que l'on peut se faire d'une langue et de ses locuteurs sont le résultat d'un processus de construction à travers différentes circonstances historiques, sociales et culturelles. En effet, les images des langues se construisent tout d'abord chez l'individu; le discours informel personnel est une manière de s'approprier la langue. Ces images des langues, constituées en imaginaires, ont été intériorisées par les membres de la société à travers le temps et par divers moyens ; elles circulent et influencent le positionnement et le comportement des individus avec leurs interlocuteurs. Nous nous intéressons ici aux images des langues dans la société mexicaine et à la façon dont elles sont perçues, représentées ou valorisées par des enseignants de français langue étrangère (FLE). Divers exemples sélectionnés seront utilisés pour montrer l'importance des images au Mexique. Les notions d'image-histoire, de hiérarchies imaginées de langues-cultures et des locuteurs seront commentées. II s'agit d'une étude qualitative réalisée dans le cadre de deux thèses de doctorat, à travers les entretiens d'un groupe d'enseignants mexicains qui explicitent leurs parcours linguistiques et laissent entrevoir leurs opinions.
\end{abstract}

Mots clés : images ; imaginaires linguistiques; FLE ; héritage ; Mexique

\begin{abstract}
Images thatwe can make aboutlanguages, cultures, and speakers result from a construction process between different historical, social, and cultural circumstances. The images are constructed individually, and then they become part of social imagination. These ideas are circulated in society in many different ways; they influence individuals' behaviors when they communicate with other speakers. In this paper, we are interested in language, culture, and speakers' images: historical images and imaginary hierarchies are exposed. This is part of a qualitative investigation done within two Ph.D. theses. We conducted interviews with young Mexican teachers to talk about their experiences in learning foreign languages and their thoughts about the languages they speak.
\end{abstract}

Key words: imaginary; foreign languages; hierarchies; legacy; Mexico 


\section{Introduction}

Le Mexique est le pays hispanophone plus grand de l'Amérique latine, il comporte environ 120 millions d'habitants. II y a, d'une part, des contacts entre l'espagnol et les langues indigènes et, d'autre part, des contacts avec les langues étrangères. Les contacts de langues peuvent avoir des conséquences sociolinguistiques parmi certains groupes, par exemple la diglossie, le déplacement des groupes linguistiques minoritaires vulnérables vers d'autres territoires, ou la mort d'une langue quelconque. Au niveau individuel, il s'agit plutôt de situations de bilinguisme ou de plurilinguisme. Il y a aussi des conséquences psychosociales, qui touchent à des questions identitaires ou bien à la conscience linguistique des individus. Il peut aussi y avoir des conséquences communicatives, telles que l'incommunication ou bien la création de stratégies pour résoudre des problèmes de communication. Enfin, il existe des conséquences politico-linguistiques, qui touchent surtout à la législation, l'éducation, les médias et la culture (Zimmerman, 2004). Partout au Mexique, on peut trouver des communautés fragilisées. La vulnérabilité linguistique se manifeste de plusieurs manières et à différents degrés; elle est en relation avec les croyances et les idéologies individuelles. Au Mexique, il existe un mépris pour l'Indigène, mais aussi pour les Espagnols en tant que conquérants. Paradoxalement, les Mexicains constituent un peuple métis né de la colonisation espagnole au XVI siècle. À l'heure actuelle, le métissage ne correspond pas uniquement aux métis biologiques, mais relève plutôt du métissage culturel (Zimmerman, 2004). Ainsi, la plupart des Mexicains sont métis. Les Indigènes voient chez les métis des traitres qui ont du sang espagnol ; et ceux qui se « sentent » Espagnols et qui déclarent ne pas être indigènes voient le métis comme inférieur à cause de son sang indigène, mais renient aussi la langue espagnole (Zunzunegui, 2013). Je me suis donc demandé si le «mépris » pouvait se rapporter aux langues, aux locuteurs. Est-ce que cela crée une survalorisation des langues étrangères?

D'une part, l'Institut national des langues indigènes (INALI, Mexique) dit qu'un locuteur sur 5 se reconnait comme faisant partie d'une communauté indigène mexicaine. Ce qui correspond à 21,5\% de la population totale du pays (INEG| ${ }^{1}$ ). D’après cette information, 6 Mexicains sur 100 parlent une des 68 langues indigènes ou bien une variante linguistique nationale; ce qui représente 7382785 personnes qui parlent une autre langue que l'espagnol. 51,3\% sont des femmes et 48,7 \% sont des hommes ${ }^{2}$.

D’autre part, il y a au Mexique des contacts avec des langues étrangères. D’après une enquête mexicaine ${ }^{3}$, les six dernières années, le pourcentage d'adultes parlant une langue étrangère au Mexique a augmenté de $9 \%$ à $13 \%$. Les Mexicains de moins de trente ans parlent plus de langues étrangères que ceux âgés de plus de cinquante ans. Ce qui veut dire que les nouvelles générations de Mexicains

1. Information disponible sur www.inegi.org.mx/. [Page consultée le 20 octobre 2020.]

2. Information disponible sur www.inali.gob.mx/. [Page consultée le 20 octobre 2020.]

3. Information en ligne sur www.totaluni.com/archivos_articulos/Mexicanos_y_los_Idiomas_59.pdf. [Page consultée le 10 novembre 2020.] Traduction personnelle. 
ont davantage la connaissance d'une langue étrangère. La formation et le niveau socioéconomique sont en relation avec l'apprentissage d'une langue étrangère. $38 \%$ des participants de l'enquête qui étudient en licence (c'est-à-dire font des études à l'université) ont déclaré la pratique d'une autre langue, tandis que $33 \%$ des participants de haut niveau social ont dit parler une autre langue. L'apprentissage d'une autre langue est plus probable en ville (16\%) qu'à la campagne (4 \%). La population de la région nord déclare plus souvent parler une autre langue (23\%) que celle de la région centre du pays (15\%).

L'objectif de cet article est de présenter un panorama de la diversité linguistique et culturelle mexicaine, ainsi que d'exposer la problématique actuelle de la situation mexicaine. À travers quelques images « héritées » et quelques extraits faisant partie des témoignages, j'essaierai de mettre en évidence certaines « hiérarchies imagées » sur les langues-cultures et les locuteurs. Les hiérarchies imagées (Torres-Castillo, 2018: 125) sont des représentations individuelles. Elles sont inscrites dans des jugements de valeur, des croyances et dans les manières de faire et de dire (Amossy et Herschberg, 1997).

Il existe plusieurs idées reçues à travers le temps sur les langues au Mexique, ainsi que quelques stéréotypes autour des Indigènes, des Européens ou des Mexicains. Une communauté a un ensemble de formules stéréotypées adaptées à différentes circonstances préétablies. La culture de la communauté est inscrite dans la langue, c'est elle qui émet des valeurs symboliques de cette culture (Gschwind-Holtzer, 1981). Le stéréotype est une idée restant ancrée dans la mémoire collective, il est aussi générateur d'une idée sur la communauté linguistique d'appartenance du locuteur. Au Mexique, on peut constater que le regard sur l'étranger a changé à travers le temps, mais que le regard péjoratif envers les Indigènes est resté ; d'ailleurs ces locuteurs deviennent vulnérables, car aux yeux de nombreux Mexicains, les Indigènes continuent d'être des sauvages, des païens, des gens sans culture ni éducation, en bref des gens non civilisés. Saenger et Yurén (2006) présentent des cas d'enseignants mexicains qui, d'après elles, adoptent une attitude de confiscation de l'altérité. Cette façon de comprendre l'autre, de l'infantiliser, est une preuve de condescendance et correspond à une fausse compréhension de l'autre. Saenger et Yurén (2006) concluent qu'il prévaut, parmi les enseignants mexicains participant à leur enquête, une attitude d'ouverture acculturante qui désavoue leur propre identité.

Les hiérarchies imaginées au Mexique vont donc opérer dans au moins deux sens. D’une part, les Indigènes ou ceux qui s'y identifient de par leurs racines indigènes, se sentiraient inférieurs devant ceux qui n'ont pas les mêmes identifications ou les mêmes caractéristiques ethniques. D’autre part, ceux qui s'identifient en tant qu'occidentaux regarderaient de travers les Indigènes et tous ceux qui auraient des traits indigènes. D'ailleurs, Villoro (2005) considère que pour un individu métis comme le Mexicain moyen, il y a toujours un « je » indigène qui, parfois, n'arrive pas à s'exprimer car l'être indigène est indissoluble de son être métis. 


\section{Méthodologie : les images des langues et des locuteurs}

Après plusieurs lectures concernant l'histoire du Mexique, j'ai décidé de réfléchir au contact des langues dans une approche historique. Pour ce faire, j'ai travaillé en tant qu'« insider », c'est-à-dire comme chercheuse partageant plusieurs références culturelles et sociales du contexte mexicain avec les autres enseignants mexicains. Je suis consciente que, parfois, les « étrangers ne puissent pas comprendre nos fatigues et nos poussières ${ }^{4}$ », non pas par défaut du regard, mais plutôt à cause d'une vision différente des choses. Certes les images sont des représentations, mais j'ai choisi la notion d'image car je la considère comme plus appropriée dans le contexte mexicain. Le Mexique est un pays de mythes et de légendes où, depuis les temps anciens, « les images pouvaient se lire, et de ce fait réduire des mots, il y avait un discours pictural qui fonctionnait en parallèle au discours oral, on pensait aussi à travers des images » (Johansson, $2014: 61)^{5}$. L'image ou les images que l'on peut se faire d'une langue et de ses locuteurs sont le résultat d'un processus de construction à travers différentes circonstances historiques, sociales et culturelles (Torres-Castillo, 2018). Ces images des langues sont constituées d'imaginaires « fruit[s] du discours ambiant » (Dabène, 1997: 19). Les images des langues correspondent souvent à des images collectives qui « sont transmises par tradition » (Gülich, 1997 : 36). On peut considérer que les images des langues sont un ensemble de croyances, c'est-à-dire ce que l'on croit savoir des langues et des locuteurs.

Le choix d'explorer les images sur les langues à travers la rencontre entre Malinche et Cortés au XVI siècle m'a permis de considérer certains des aspects historiques qui constituent l'héritage imaginaire des enseignants mexicains. D'ailleurs, les images sont vues ici comme des « émergences d'une expérience antérieure » (Wunenburger, 2003: 57), une sorte d'expérience revécue par les individus.

\subsection{Les statuts formel et informel des langues au Mexique}

D’après Dabène (1997), les langues existent dans deux espaces. Le premier espace est représenté par l'usage concret qu'une société fait des langues. Il est constitué par les dispositions officielles à caractère juridique qui régissent l'emploi et l'enseignement des langues. Cet espace est aussi constitué par les politiques linguistiques qui encouragent l'utilisation d'une langue ou qui permettent aux locuteurs l'utilisation d'une langue particulière dans un contexte précis. Le deuxième espace est celui qui se réfère au discours des membres du « corps social » (étudiants, institutions, familles et enseignants).

Le statut informel des langues se rapporte à la subjectivité de l'individu, qui s'approprie différemment la ou les image(s) de langues et des locuteurs. Pour comprendre cette « réalité » imaginaire, je me suis basée notamment sur trois aspects de l'épistémologie multiculturelle de Semprini (1997).

4. Adaptation personnelle du texte de Garro (2003: 350).

5. Traduction personnelle. 
- «La réalité est une construction » (p. 59). C'est-à-dire que pour comprendre les statuts informels, il faut tenir compte des histoires personnelles des Mexicains.

- «Les interprétations sont subjectives » (p. 59). Plusieurs valeurs transmises par héritage culturel sont des constructions intersubjectives. Les apprenants de français possèdent diverses hiérarchies imaginaires. Ce sont des interprétations subjectives qui, parfois, ne reflètent pas la réalité partagée par tout le monde.

- «Les valeurs sont relatives (p. 59). Pendant plusieurs années, dans les formations de FLE, le public mexicain s'est vu attribuer une problématique commune et homogène. Dans les ouvrages de didactique du français, on a tendance à classer les apprenants de FLE comme «non-natifs », avec des caractéristiques communes à tous ceux qui appartiennent à cette catégorie absolue. Cependant, plusieurs valeurs accordées aux langues, aux locuteurs, à l'autre, sont enracinées dans leurs histoires personnelles (Torres-Castillo, 2018).

À travers certaines catégories présentées lors de cette étude, j'ai essayé de découper cette « réalité » et j'ai constaté qu'elle était parfois construite sur des édifices imaginaires.

\subsection{L'étude}

Cette étude de terrain a été en partie réalisée en 2011 et 2012 auprès de jeunes enseignants de français langue étrangère au Mexique. II s'agit d'enseignants formés dans un programme mexicain de licence ou de master en didactique du français dans des universités publiques mexicaines (l'Université de l'État de Mexico et l'Université de Veracruz à Xalapa). Il s'agit d'une étude qualitative dans le cadre de deux thèses de doctorat. Une partie du corpus est constituée d'entretiens semi-dirigés transcrits. Des questionnaires ont été remplis par les étudiants du master en didactique du français de Xalapa en 2012. À ces données s'ajoute l'observation des enregistrements vidéo de diverses classes de français langue étrangère. Parfois, les participants ont répondu en espagnol : dans ce cas, les entretiens ont été traduits en français.

Comme les données ont été recueillies en 2011 et 2012, on peut penser qu'une mise à jour des résultats devrait être envisagée. Cependant, comme il s'agit d'imaginaires collectifs, nous pensons que les idéologies perdurent, qu'elles ne changent pas du jour au lendemain. Les chiffres concernant les enseignants de français ont probablement évolué ces huit dernières années. Ajoutons que plusieurs images sont inscrites dans l'histoire du Mexique. La Conquête du Mexique est, encore aujourd'hui, un thème d'actualité au Mexique. Un événement autour de cette thématique a eu lieu en 2019 lors du 500 anniversaire de l'arrivée de Cortés au Yucatan. Le président du Mexique a demandé au roi d'Espagne et au pape de s'excuser ${ }^{6}$. La prise en compte de cet évènement historique a permis une approche innovatrice. L'étude documentaire s'est faite à travers ce que j'ai nommé « les images-

6. Information en ligne sur https://cubasi.cu/es/noticia/mexico-presidente-pide-disculpa-de-la-iglesia-por-conquista. [Page consultée le 10 novembre 2020.] 
histoire » (Torres-Castillo, 2018: 146). Au fil du temps, ces images ont acquis un caractère de plus en plus important, elles ont été plusieurs fois associées à la magie et à la religion.

\subsubsection{Les images-histoire au Mexique}

Dans l'Antiquité, au Mexique, l'image servait de moyen de communication. Le monde était exprimé à travers des images, car on voyait au travers d'un schéma visuel les personnes ou les objets du monde réel. Cependant, « les Indiens ne partageaient pas la conception espagnole de l'image (...) », « la peinture indigène n'est pas une image à proprement parler. C'est pour une part, un mode de communication graphique soumis à une logique de l'expression et non pas au critère d'une imitation réaliste jouant un redoublement » (Gruzinski, 1990 : 85). De plus, « les codex ont été conçus pour être lus, les codex nahuas maintenaient la mémoire structurée (...) La configuration de l'image ne se soumettait pas à la "tyrannie" des mots mais elle présentait du sens avec une sémiologie propre » (Johansson, $2004: 189)^{7}$.

Les codex mésoaméricains sont élaborés dans une espèce de papier fait à base de liber de ficus nommé amatl (en nahuatl). Les codex mexicains ont été pour la plupart détruits, ou se trouvent dans des collections privées inaccessibles au grand public. D’autres codex ont été produits après l'arrivée des Espagnols au Mexique. Ces codex ont été principalement utilisés pour l'évangélisation. Cependant, dans certains cas, les codex ont servi à des moines comme moyen de préserver cette tradition expressive. Les codex ont été peints par les Indigènes car il existait une tradition de scribes qui racontaient des histoires, des légendes à travers des images. Ces documents ont été faits par des Indigènes sous la « direction » des moines espagnols. Il faut notamment mentionner l'Histoire générale des choses de la Nouvelle-Espagne (Sahagun et Jourdanet, 1880).

Les images qui nous intéressent particulièrement ici montrent les contacts de langues du Mexique. Ce sont celles dont Malinche et Cortés sont les personnages centraux. Il est important de considérer que dans les images-histoire mentionnées ci-dessous, les couleurs ont de l'importance parce qu'une même couleur pouvait signifier des symboles collectifs différents. Les couleurs sont d'ailleurs vues d'une façon différente selon le contexte : Wunenburger (2003 : 64) signale que «les couleurs, par exemple, se voient dotées, dans toutes les cultures, de valeurs affectives et intellectuelles analogues (...)».

\subsubsection{La langue étrangère, un instrument de pouvoir}

El lienzo de Tlaxcala a des caractéristiques hybrides. Ce document présente le récit en détail de la Conquête du Mexique, il montre la vision des Indigènes. Les images contenues dans ce document forment un inestimable livret visuel. C'était aussi un outil pour ceux qui avaient participé à la Conquête du Mexique. Ils pouvaient réclamer de se faire payer pour leurs services à la couronne espagnole (Bueno-Bravo, 2010). Ce document peint par des Indigènes au XVIe siècle a été fait en trois originaux

7. Traduction personnelle. 
qui se sont perdus. Une copie en a été faite par l'association Mesolore ${ }^{8}$. Cette reproduction utilise la lithographie imprimée en 1892 par Chavero.

Les images-histoire présentées ici sont extraites du codex El lienzo de Tlaxcala9. Sur ce document figure le soutien du peuple tlaxcalteca à Cortés pendant la Conquête du Mexique (Nuñez-Rodriguez, 2012). Malinche apparait dans plusieurs scènes; elle est toujours placée comme médiatrice. Alors que les femmes étaient habituellement représentées assises, « elle est peinte debout, l'équivalent de la liberté d'une prostituée » (Thomas et Villeneuve, 2011: 368). On la voit à côté de Cortés; dans d'autres images on la voit transformée (Fig. 1), car elle porte des chaussures espagnoles qui contrastent avec les habits traditionnels des femmes indigènes. Cette image voulait probablement exprimer le fait que Malinche parlait espagnol (Torres-Castillo, 2018).

Figure 1 : El lienzo de Tlaxcala (Image 19/86) ${ }^{10}$

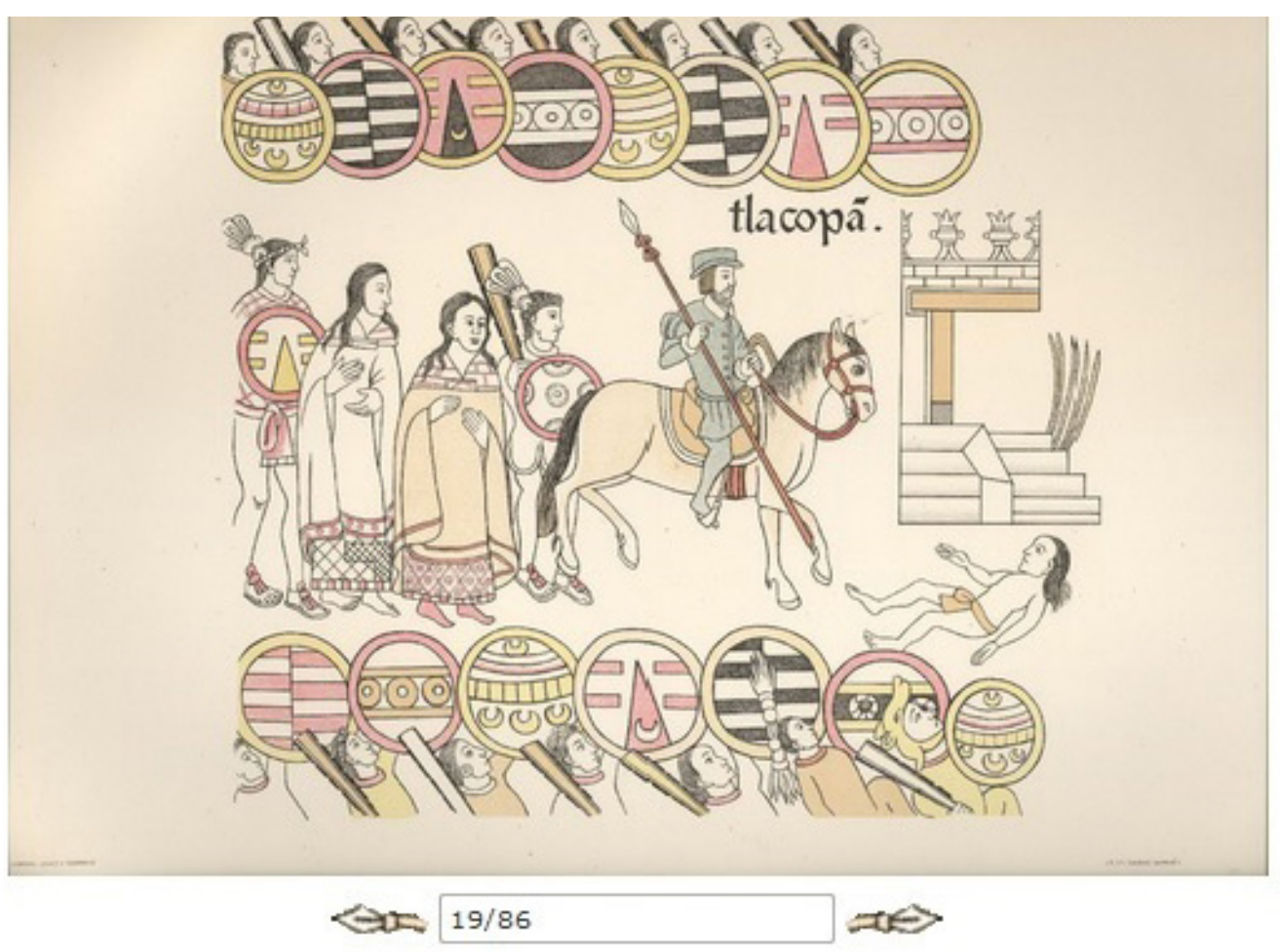

8. Un aperçu de cette copie accompagné d'explications est disponible sur www.mesolore.org/tutorials/learn/19/ Introduction-to-the-Lienzo-de-Tlaxcala. [Page consultée le 10 novembre 2020.]

9. El lienzo de Tlaxcala, explicación de Alfredo Chavero 1892. Information disponible sur http://marcofabr.blogspot. fr/2011/05/explicacion-del-lienzo-de-tlaxcala-por.html. [Page consultée en novembre 2016.]

10. Source : www.mesolore.org/tutorials/learn/19/Introduction-to-the-Lienzo-de-Tlaxcala. 
Dans ce document, Malinche est "présentée d'une taille plus grande » (Fig. 2), parfois les seuls qui détiennent le glyphe représentant la " parole » sont Cortés et Malinche. "Dans une autre image, Cortés est tourné vers Malinche, presque en tant qu’observateur »(Brotherston, 2013 : 11).

Figure 2: El lienzo de Tlaxcala (Image 6/86) ${ }^{11}$

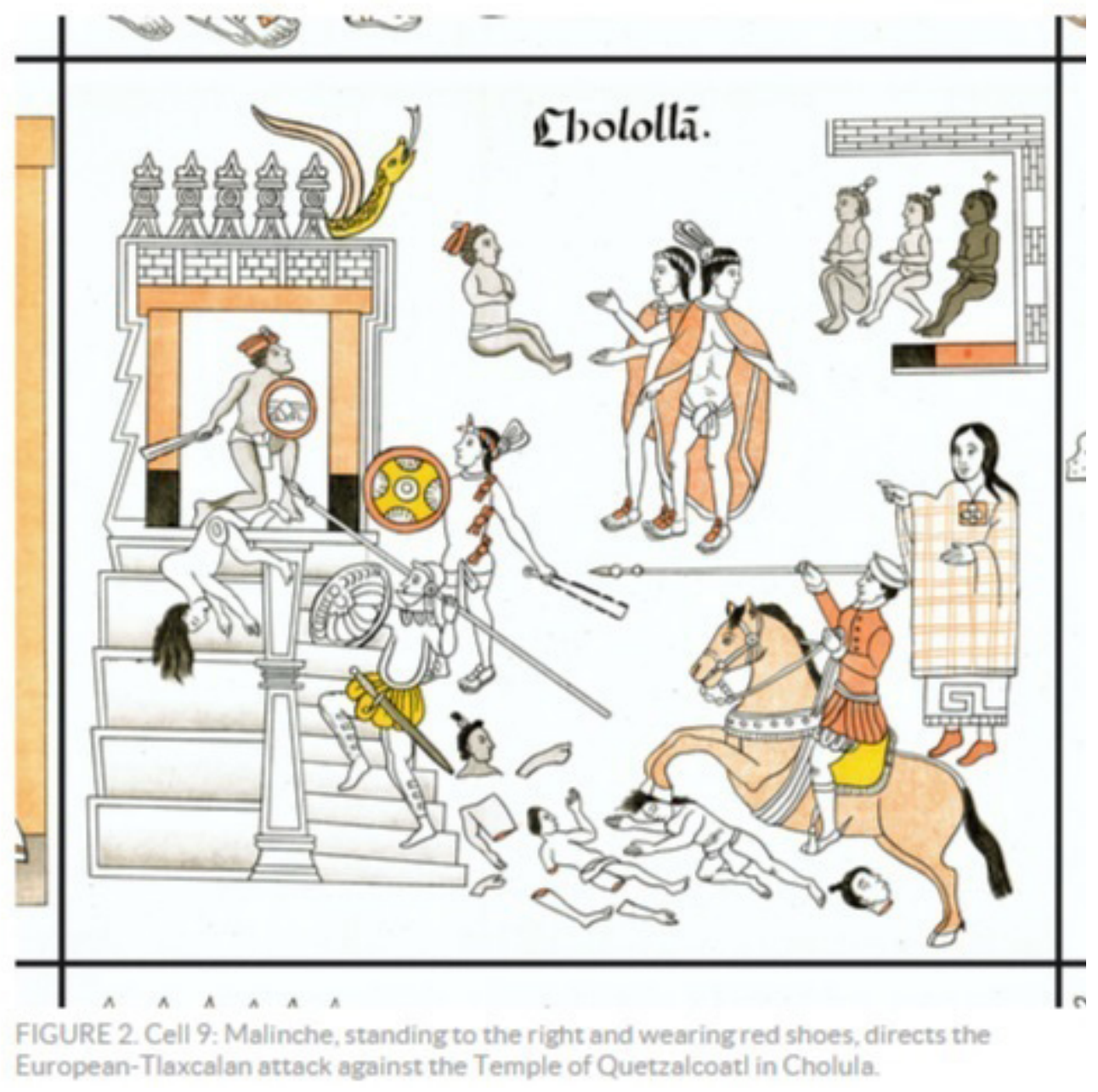

Dans cette image, on peut aussi apprécier la participation de Malinche en tant que médiatrice culturelle. Elle se situe entre plusieurs locuteurs de langues distinctes (nahuatl-maya-castillan).

Même si le mythe autour de Malinche et Cortés est toujours vivant au Mexique, ces deux personnages provoquent encore des controverses. Pendant de nombreuses années, cette femme a été présentée dans les livres d'histoire comme la maitresse de Cortés et la complice de la victoire sur les Mexicains (Bartra, 2013). D’après Zuñiga (2003), les « mémoires historiques » héritées via des écrivains, des chercheurs, conscients ou pas, continuent d'alimenter la domination à travers des mythes.

11. Source: www.mesolore.org/tutorials/learn/19/Introduction-to-the-Lienzo-de-Tlaxcala. 


\section{Diversité linguistique et contacts de langues au Mexique}

Divers contacts entre les langues européennes et les langues indigènes se sont déroulés depuis les premières rencontres, lors de la conquête du Mexique au XVI e siècle. Actuellement, il existe des communautés diglossiques partout dans le pays, par exemple dans la zone du Yucatan ou celle du centre du pays (Zimmerman, 2015). L'espagnol parlé actuellement est le résultat de plusieurs contacts de langues ayant eu lieu au fil du temps au Mexique en tant que colonie espagnole. L'espagnol a intégré plusieurs mots du nahuatl et d'autres langues indigènes (Zimmerman, 2004). Actuellement, l'enseignement de l'espagnol au Mexique suit trois grandes orientations : l'espagnol enseigné aux enfants et adultes scolarisés au Mexique ; l'espagnol enseigné en tant que langue étrangère, destiné aux étrangers non hispanophones; et l'espagnol enseigné aux Indigènes (Torres-Castillo, 2018).

L'anglais en tant que langue étrangère a une grande influence au Mexique, c'est la langue du voisin du nord, les États-Unis. On peut comparer le nombre de locuteurs de langues indigènes, qui s'élève à peine à 7 millions de personnes (connues), aux « 9,5 millions de Mexicains de plus de 18 ans qui déclarent parler anglais. Dans le nord du pays, 1 adulte sur 5 dit parler cette langue, tandis que dans le sud du pays, 1 sur 25 seulement la parle ${ }^{12}{ }^{2}$.

En comparant le nombre de locuteurs de langues indigènes actuel avec celui des locuteurs de l'anglais, on pourrait dire qu'il est normal de trouver un grand écart, puisque l'anglais est une langue internationale avec beaucoup d'influence dans le monde. Mais il faut prendre en considération que le nahuatl a été un temps la langue « officielle » de l'ancien Mexique : elle a été pendant environ 200 ans, entre 1521 et 1771, une langue privilégiée maintenant le fonctionnement de la société coloniale. L’espagnol était plutôt considéré comme la langue étrangère, celle du colonisateur.

Puis le nahuatl a cessé d'être admis dans la Nouvelle-Espagne par un décret royal. Entre 1771 et 1821, l'espagnol est devenu la langue principale de cette colonie (Barriga et Butragueño, 2015). C'est seulement en 2003, avec la création de la Loi des droits linguistiques des communautés indigènes au Mexique, qu'une partie de la population mexicaine parlant une autre langue que l'espagnol a été reconnue. Cependant, la reconnaissance est établie selon un caractère formel et non pas sur le statut informel. Au Mexique, on risque de continuer à croire à cette « vérité historique » présente dans l'imaginaire collectif, selon laquelle l'infériorité du Mexicain devant l'étranger serait réelle. Et par conséquent, on continuera à entretenir le mépris pour les langues et les locuteurs indigènes et l'exaltation de la langue étrangère et de l'étranger.

12. Information disponible en ligne sur : www.totaluni.com/archivos_articulos/Mexicanos_y_los_Idiomas_59.pdf. [Page consultée le 10 novembre 2020.] Traduction personnelle. 
Actuellement, l'offre de cours de langues indigènes dans les centres de langues ou universités est moins importante que celle de cours d'anglais, par exemple. On pourrait même penser que les langues indigènes sont des langues étrangères et lointaines pour certains Mexicains. On peut souvent entendre dire, de façon populaire, « que ces langues ne servent à rien ».

L'enseignement de langues étrangères au Mexique est notamment composé de celui de la langue anglaise, suivi par celui du français. Ces deux langues sont arrivées formellement au XIXe siècle, notamment après l'indépendance de l'Espagne.

Le français a été introduit au Mexique grâce aux contacts commerciaux et à la présence française dans ce pays. Les communautés françaises au Mexique étaient notamment formées d'instituteurs français qui se sont installés au nord du pays. Ces enseignants sont venus pour satisfaire la demande de «bons enseignants » provenant de la classe aisée mexicaine du XIXe siècle (Hernandez, 2005). La bonne qualité de l'enseignement en général, et pas exclusivement celui des langues, était associée et réservée aux étrangers. Le français a été introduit au Mexique comme langue « savante ». On croyait que les connaissances étaient mieux transmises par ces instituteurs étrangers, et que la formation des enfants était ainsi entre de meilleures mains (Torres-Castillo, 2018).

Au Mexique, la diffusion du français langue étrangère a été fortement caractérisée par l'offre de cours de l'Alliance française, ouverte en 1886, et celle de l'IFAL (Institut français d'Amérique latine),

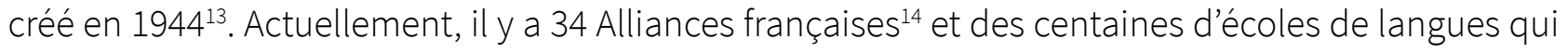
enseignent le français parmi d'autres langues. En 2017, l'ambassade de France au Mexique estime à 250000 le nombre d'étudiants de français sur le territoire mexicain. «L'essor de la langue française au Mexique » est évoqué par Riba (2011) à travers le nombre de candidats aux diverses certifications construites sur le Cadre européen commun de référence pour les langues (CECRL) : en 2010, " 10600 candidats se sont présentés aux épreuves et ont fait du Mexique le $5^{\text {e }}$ pays du monde en termes de nombre de candidats » (p. 48). Les apprenants de français mexicains vivent au moins entre deux cultures et deux langues qui s'entremêlent (Torres-Castillo, 2015). D'ailleurs, la prise en compte d'autres facteurs que la seule compétence linguistique en français, par exemple la diversité des parcours professionnels des apprenants (Goi et Torres-Castillo, 2013), apporte de l'information sur les statuts informels des langues.

\section{Les hiérarchies imaginées}

Les hiérarchies peuvent exister dans des contextes différents et prendre différentes formes. TorresCastillo (2018) mentionne les hiérarchies sociales : le prestige et la légitimité. Dans le cas du Mexique, ces hiérarchies peuvent très bien se présenter à travers le prestige de la langue parlée. Les locuteurs

13. Information disponible sur https://ifal.mx/quienes-somos/qui\%C3\%A9nes-somos. [Page consultée le 25 septembre 2020.]

14. Information disponible sur https://alianzafrancesa.org.mx/ [Page consultée le 25 septembre 2020.] 
d'une langue indigène sont souvent considérés comme des gens sans culture et sans éducation. Parler une langue indigène au Mexique est signe de pauvreté. A contrario, les locuteurs d'une langue étrangère peuvent être considérés comme des individus qui ont plus de prestige social ou appartenant à une élite.

Les hiérarchies culturelles sont établies sur les traditions d'une culture donnée, la culture française est souvent considérée comme dominante ou «meilleure»; ces constructions hiérarchisées peuvent surgir à travers l'origine culturelle ou religieuse.

Les hiérarchies géopolitiques, en relation avec l'origine géographique de naissance, forment une croyance politique; il en va ainsi de la supériorité du locuteur natif d'une langue. Pour les locuteurs du français, on peut utiliser l'exemple du français de la Touraine, qui est réputée parmi les Français comme « la région où l'on parle le meilleur français en France ». À ce titre, Castellotti (2006 et 2011) mentionne les idées connotées par l'accent de Tours : les témoins disent qu'il s'agit d'une « prononciation impeccable », d'une «prononciation parfaite », de la « meilleure prononciation », de l'endroit où les étudiants peuvent apprendre « sans accent », où il y a «l'accent pur », le « vrai accent », ou «l'accent authentique».

Extrait de l'entretien de Minerva, lorsqu'elle parle de l'accent de son enseignante française, qui parle en espagnol ibérique (Torres-Castillo, 2018 : 360) :

8-B Comment a été ton expérience d’apprentissage (du français) ?

9-M ç’a été intéressant et en même temps difficile car la professeure (française) que j’ai eue pendant le premier semestre parlait très peu espagnol et en plus c'était de l'espagnol ibérique, quand elle devait nous donner des exemples ou nous expliquer quelque chose, c'était difficile pour elle de le faire en espagnol, alors elle commençait à parler en français, elle se servait des gestes et des images pour nous expliquer.

Cette même participante a répondu ainsi à la question qui demandait pourquoi elle n'enseignait pas l'espagnol ${ }^{15}$ :

Pour moi, devenir enseignante de français est une très belle expérience, mais en même temps est un défi car il ne s'agit pas seulement d'enseigner l'usage de la langue mais aussi de la culture de France et des pays francophones. J'ai eu l'occasion d'enseigner l'espagnol dans le centre de langue et même s'il s'agit de ma langue maternelle, je ne me suis pas sentie à l'aise, en tout cas pas avec la même aisance que j'ai quand je fais des cours de français.

L'enseignante qui déclare ceci dit aussi avoir été conquise par la musicalité de la langue française (Torres-Castillo, 2018 : 355). Pour Minerva, les sons d'une langue exprimés à travers les accents sont des traits importants pour établir ses propres hiérarchies de valeurs.

15. Traduction personnelle. 
Les hiérarchies sont nées entre colonisés et conquérants; par exemple des hiérarchies naturelles ou biologiques, fondées sur l'apparence physique et « la race ». Il existe également d'autres hiérarchies construites sur l'ethnie ou sur la capacité cognitive; et aussi des hiérarchies de genre, organisées autour des différents rôles entre les hommes et les femmes. Plusieurs de ces hiérarchies peuvent être clairement mises en évidence à travers les exemples présentés ci-dessus (les images-histoire).

Pendant la colonie espagnole, le pape a autorisé les mariages des Espagnols et des Indigènes, avec l'idée que ce métissage permettrait la substitution d'une ancienne âme par une nouvelle (Torres-

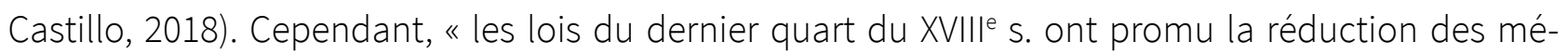
langes raciaux et surtout, ont essayé de garder les éléments de distinction des groupes dominant ${ }^{16}{ }^{\text {}}$ (Gonzalbo, 2012: 125).

Au Mexique, «la plupart des métis» sont nés avec le stigmate de l'abandon paternel (Ramírez, 2005: 239), avec l'image d'une mère indigène soumise qui a juste satisfait le besoin sexuel d'un homme. L'image du père est associée à la force et à l'infidélité acceptée par la société. Les enfants des métis ont donc grandi avec des images stéréotypées des femmes et des hommes. Ramos, cité par Uranga (2005), dit que c'est au moment de la Conquête que le complexe d'infériorité (présupposé chez les Mexicains) est né.

Les hiérarchies professionnelles et d'apprentissage sont tantôt jugées en termes de performance dans le métier, tantôt construites autour du prestige de la formation professionnelle ou de l'expérience en enseignement et la transmission d'un certain savoir. Elles peuvent être présentées à travers les différentes images qu'on se fait du bon enseignant du français. On pense souvent que le locuteur natif est plus compétent en tant qu'enseignant et que le locuteur non natif est un mauvais enseignant, car il a un accent et ne maitrise pas parfaitement le français.

En ce qui concerne les hiérarchies en relation avec les capacités ou les aptitudes dans l'enseignement des langues autour des compétences à enseigner, on privilégie parfois uniquement la compétence orale et on évalue très facilement les compétences d'une personne à travers la présence ou pas d'un accent étranger.

\subsection{Comprendre l'autre à travers des hiérarchies imaginées}

Charaudeau (1995) explique comment une « force souterraine » nous entraîne à essayer de comprendre l'autre. D'une part, on se découvre différent devant l'autre et on se découvre « incomplet, imparfait, inachevé » (p. 16). D’autre part, on se construit des hiérarchies imaginées avec lesquelles on cherche à savoir si l'autre est supérieur ou s'il représente une menace pour soi. Le rejet de l'autre est plutôt exprimé lorsqu'on le voit comme une menace.

16. Traduction personnelle. 
Les préjugés, les clichés ou les stéréotypes concernant l'autre sont plutôt vus comme une nécessité pour se protéger de l'autre, « ils constituent d'abord une protection, une arme de défense contre la menace que représente la différence » (Charaudeau, 1995 : 17). La rencontre avec l'autre, vue par Charaudeau, fait surgir un paradoxe chez l'individu (Torres-Castillo, 2018). L'autre serait jugé négativement (trop froid, rationnel ou agressif), car on se considère comme « meilleur », car on est persuadé que l'on est exactement son contraire. Nous serions convaincus que « nos normes sont les seules possibles » (Charadeau, 1995 : 17), l'autre serait alors en défaut car il ne suit pas nos normes. À titre d'exemple, imaginons qu'un individu juge qu'il parle correctement le français : c'est à la fois en fonction de ceux qu'il voit comme un modèle idéal (tel celui du locuteur natif) auquel il cherche à s'assimiler, et de l'autre qui devient alors celui qui ne maitrise pas suffisamment bien la langue française.

Il apparait évident que les diverses hiérarchies empêchent les bonnes relations avec les autres, " parce qu'il s'agit plutôt de constructions négatives qui changent la réalité et dès lors la façon d'agir. Peut-être qu'elles empêcheront de se surpasser ou de s'améliorer » (Torres-Castillo, 2018 : 129). Les hiérarchies imaginées deviendraient des obstacles à la découverte de l'autre ainsi que des chaines de domination plus ou moins acceptées. Les hiérarchies imaginées sont à la base des raisons par lesquelles s'expliqueraient plusieurs comportements glottophobes et situations de vulnérabilité linguistique, qui atteignent tous les locuteurs à un moment donné. S'agissant des images individuelles, les hiérarchies imaginées sont des façons de voir la réalité et surtout des conditionnements dans l'approche de l'autre.

\subsection{Hiérarchies imaginées de locuteurs}

Charaudeau (1995) et d'autres chercheurs ont remarqué que la valeur positive ou négative varie selon la situation du groupe qui répond. Ils ont donc utilisé la psychologie sociale, notamment pour expliquer les catégories sociales et les rapports de domination. Il y a une certaine tendance à l'assimilation (des « inférieurs » aux « supérieurs »). Les individus s’approprient des qualités positives de l'autre groupe prestigieux (« inférieurs » vers les « supérieurs»), c'est ce que Charaudeau appelle « assimilation positive ». Mais il existe aussi une « assimilation négative », lorsque les « supérieurs » perdent des qualités positives (p. 26). "Un autre phénomène appelé "substitution" serait celui de garder ses valeurs et s’attribuer les qualités des autres, qui sont censés les perdre » (p. 27).

Prenons comme exemple ce que Gadet et Ludwig (2015) décrivent comme une « longue tradition de condescendance » (p. 47) : «La condescendance se voit dans des moqueries et des jugements négatifs sur la façon dont ces autres locuteurs s'expriment en français » (p. 45). Ceux qui se pensent supérieurs disqualifient les locuteurs à travers une série de termes « péjoratifs ». Plusieurs fois, la suprématie imaginée repose sur une supposée légitimité, sur un modèle de locuteur imaginaire. Quelques exemples sont présentés par Gadet et Ludwig (2015 : 45-46) : « Le terme baragouin (dérivés baragouiner, baragouinage) qui désigne à l'origine les Bretons parlant mal le français ». Un autre 
exemple serait celui de "petit-nègre », «terme qui désigne des variantes du français jugées dégradées ou inférieures, supposant des représentations caricaturales, dévalorisantes, racistes ». À travers ces termes, la langue parlée par ces individus est intégrée en même temps à une échelle de valeurs. Parler un « petit français », "français cassé », « français-façon » (p. 47), ou un français comme celui des Français du sud s'exprimant dans une langue française régionale et avec un accent particulier, entraîne une qualification de "campagnards ». Ces locuteurs sont en même temps caractérisés comme inférieurs car ces individus ne correspondent pas aux modèles imaginaires du locuteur. Ces situations peuvent se reproduire aussi chez les apprenants du français, ou bien chez les allophones, à qui on collera l'étiquette de «non-natif » et donc ayant des soucis de compréhension en français: peu importe leur niveau de maitrise de la langue étrangère aux yeux de différentes personnes, ils auront une image dégradée et inférieure.

Pendant la colonisation espagnole au Mexique, ceux qui se sentaient « supérieurs » ont agi avec la même condescendance envers les Indigènes. Leur façon de traiter les Indigènes se justifiait sur la base de la foi chrétienne, la manière de s'adresser aux autres était donc particulière car il fallait respecter certains protocoles. La condescendance envers les Indigènes s'est en quelque sorte muée en domination extrême, justifiée par le vasallaje et l'encomienda : par le prétexte d'aider les Indigènes à se corriger et à devenir des « personnes » civilisées.

Le Mexicain «modèle » est décrit à partir de plusieurs critères. II s'agit de traits caractéristiques qui n'échappent pas à l'œil des Mexicains. Physiquement, il a la peau brune, bronzée, les cheveux très raides. Quelquefois, les traits physiques sont plus indigènes ou bien plus européens. Dans ce cas-là, on peut trouver des distinctions et même de la discrimination envers ceux qui ont des traits indigènes. L'apparence physique correspond aussi à la façon de s'habiller et même de se tenir. Souvent, on associe un comportement à la « race».

Alors que tous les habitants du pays sont actuellement des métis, il existe du mépris et de la distinction. Il y a encore aujourd'hui des expressions qui décrivent des comportements autrefois associés aux Indigènes. Se faire appeler « indio » ou «naco » est une insulte qui implique le manque de goût et d'éducation. L'image des indios est particulièrement distincte dans quelques secteurs de la population mexicaine.

L'indio n'a pas de culture générale, il vit encore attaché à des habitudes et des traditions, et par des liens avec des superstitions. L'indio a de l'amour pour la terre, il n'a pas beaucoup d'émotions, il a seulement des émotions pour ce qui pendant longtemps a été sa passion [...] $\left(\right.$ Chavez, 2002:32) ${ }^{17}$.

En Nouvelle-Espagne, les femmes étrangères (peninsulares) étaient valorisées, elles s'occupaient des activités religieuses et civiques, mais pas de leurs enfants, «criollos». Pour s'occuper des enfants, il y avait les « Marias de a peso », des femmes indigènes. La « nana », femme indigène, était dévalorisée

17. Traduction personnelle. 
par sa condition indigène (Ramirez, 2005). Les premières années dans la Nouvelle-Espagne étaient sous la coupe des criollos (Espagnols nés en Amérique). Les Indiens « ont été exploités par tout le monde, par le propriétaire criollo et par le fonctionnaire métis» (Villoro, 1996 : 212).

Le modèle du locuteur fantasmé est celui du locuteur « natif », habituellement défini comme « sujet parlant sa langue maternelle et considéré à ce titre comme l'ayant parfaitement intériorisée, donc capable de jouer le rôle d'informateur » (Galisson et Coste, 1976 : 366). Il y a plusieurs choses à dire autour de ce modèle : diverses hiérarchies imaginaires peuvent surgir à travers cette notion. En fait, la promotion du monolinguisme à travers un modèle fantasmé serait plus populaire que celle du plurilinguisme.

Extrait de la réponse de Lorena à la question n 3 du questionnaire de Xalapa, lorsqu'on l'interroge sur sa posture en tant qu'enseignante «non native»:

Il y a des personnes qui pensent que l'enseignement de la L2 est meilleur quand on apprend avec un natif. Moi, je dis que les natifs ne sont pas toujours la meilleure option. Bien qu'ils aient la maitrise de la langue, dans plusieurs cas ils ne sont pas formés à l'enseignement et ils n'arrivent pas à expliquer quelques aspects de grammaire/afin que les apprenants comprennent clairement les règles d'usage de la langue/++D'autre part connaitre la langue des apprenants donne des avantages/parce que tu sais quelle est la raison principale à l'origine de leurs fautes/le fait d'être passé par la même expérience te donne des outils pour pouvoir les aider. (Torres-Castillo, 2018 : 349)

Contrairement à ce que plusieurs enseignants mentionnent pendant l'enquête, l'enseignant « natif » peut lui aussi expérimenter des moments d'insécurité linguistique, car le «natif» n'est pas toujours sûr de lui, cela dépend de la situation d'enseignement, du niveau et de l'âge des apprenants.

Cependant, au Mexique, le locuteur « natif » du français est survalorisé, ceci grâce au statut informel des langues ancrées dans les imaginaires collectifs. Le « natif » occupe une place privilégiée dans plusieurs écoles et parmi le grand public ; la qualité de l'enseignement est plusieurs fois associé à l'accent de l'enseignant, la plupart du temps la prononciation gallo-française ou celle de « Paris » est la mieux acceptée. Les capacités et compétences pour le travail se voient réduites à des questions phonologiques, un accent imaginé est accepté en tant que norme ou comme standard linguistique. Il ne s'agit pas uniquement d'une discussion sur les pratiques de recrutement au Mexique, mais plutôt des favoritismes existants et du manque de justice sociale, celle-ci comprise comme la possibilité d'accéder à un emploi. 


\subsection{Hiérarchies imaginées de cultures}

Parmi les témoins, on a pu distinguer une certaine valorisation de la culture française, même si les témoins n'ont pas dévalorisé/méprisé leur culture d'origine. Ceci prouve l'existence des hiérarchies individuelles. Elle peut s'expliquer à travers un texte qui servait de source pour l'enseignement au Mexique. Voici la traduction personnelle de quelques extraits de l'œuvre de De la Torre (1893 : 219221)

C'est le pays des excès mais celui-ci est couvert d'une voile délicate et amusante : les femmes ont la douceur du langage qui au début dérange, mais au bout de quelque temps attire et séduit d'une façon irrésistible, elles ont une élégance et aisance extraordinaires pour porter ces habits, même s'ils sont laids et conservateurs, chacune d'elles porte ce qui est à la mode (...) En un mot, la femme française sait gagner de l'argent et elle sait aussi économiser et épargner.

Mais pour une raison étrange et malgré ces compétences, à Paris il n'y a pas de foyers, il n'y a pas de famille (...) Quand il y a des enfants ils vont habiter loin des parents ${ }^{18}$.

De la Torre (1893) présentait probablement une introduction au « savoir-vivre » d'autres pays à travers son document utilisé comme livre de textes des écoles primaires pour des filles mexicaines. Ces filles devaient apprendre ces préceptes pour pouvoir appartenir à la haute société. Il faut mentionner que les filles qui avaient accès à une formation formaient une minorité privilégiée. On peut supposer que les modèles imaginaires de l'époque étaient inspirés des Européens, car en 1893, on vivait au Mexique sous la dictature de Porfirio Diaz, qui adorait la France. De plus, le ministre des Finances était alors un Franco-Mexicain nommé José Yves Limantour. Lui et sa famille représentaient à l'époque le raffinement de la classe haute mexicaine. La culture française était alors exaltée parmi les autres nations. Il n'est donc pas très étonnant de lire que la « France est le pays civilisé par excellence, et où on peut trouver des gens élégants et qui montrent une grande aisance extraordinaire, c'est aussi le pays qui fait très attention aux bonnes manières » (De la Torre, 1893 : 219).

Par exemple, l'auteur donne aux femmes françaises l'incroyable pouvoir de séduire à travers leur façon de parler. Les femmes mexicaines et françaises sont comparées. Une certaine hiérarchie s'établit puisque la femme française a ce pouvoir parce qu'elle parle en français, tandis que la femme mexicaine n’a pas cette capacité. Le texte a été écrit par un homme, et il semble appartenir à un imaginaire masculin de l'époque. Dans le même texte, vers la fin de son récit, De la Torre fait une comparaison et une sorte de remise en question de l'importance de la famille mexicaine. Il affirme « qu'à Paris il n'y a pas de famille (p. 221) [une autre image fantasmée, car les Françaises sont-elles originaires uniquement de Paris?].

18. Extrait du texte espagnol. Traduction personnelle. 
Chez Charaudeau (1995), on peut aussi voir comment les imaginaires autour des femmes mexicaines coïncident encore avec ceux qu'on avait à l'époque coloniale, où la femme indigène était soumise par tradition. Les imaginaires concernant les femmes françaises coïncident également avec ceux utilisés dans l'éducation basique au Mexique, présentés par De La Torre.

Dans l'étude de Charaudeau (1995), les Mexicains pensent que les Français sont ou se croient supérieurs. Les femmes mexicaines pour leur part pensent que les femmes françaises sont supérieures (p. 40).

Les enseignants de français interviewés ont aussi leurs idées sur la culture française, ces idées sont inscrites dans leurs propres expériences quotidiennes avec les locuteurs de cette langue, ou bien à travers leurs vécus et aussi via les imaginaires collectifs.

(...) « La culture française est celle qui a plus apporté à l'humanité, à travers des manifestations artistiques, sociales, politiques, sportives, etc. Je considère qu'il est vraiment nécessaire que les étudiants puissent connaitre la culture française. » Exemple 5 : Extrait de la réponse à la question 2 de la participante Fany, de Xalapa, (traduction personnelle) lorsqu'elle parle de la «culture française ». (Torres Castillo, 2018 : 282)

"Le français est une langue officielle et d'une certaine manière, la langue de la culture. » Exemple 13 a : Extrait de la réponse de Viridiana à la question 1 du questionnaire de Xalapa (traduction personnelle), lorsqu'elle parle de la culture. (Torres Castillo, 2018 :293)

Ces déclarations mettent en valeur la «culture française ». Elles nous inciteraient à penser que le pays où se « produit » la « culture » « véritable » est la France, ou bien que c'est le pays où l'on s'intéresse par tradition à la «culture cultivée ». On peut faire l'hypothèse que la participante ne place pas sa culture d'origine au premier rang (Torres Castillo, 2018). Ces exemples nous permettent de voir que ces enseignants ont placé la culture française en accord avec des hiérarchies imaginées individuelles.

Parmi les participants de Xalapa, Jaime, María et Fany sont d’accord, ils pensent « qu'à travers la langue on découvre un nouveau monde ». Dans le même sens, les participants Illse, Sandra, et Viridiana pensent qu'avec « une langue on peut apprendre une culture, voyager et acquérir de nouvelles connaissances». (Torres Castillo, 2018:300)

Dans cet exemple, la langue est liée à la «culture française ». Les participants expriment leur admiration et leur enchantement pour la langue française, peut-être au détriment de leur propre langue et culture. Peut-être que la découverte n'existe pour eux qu'à travers le dépaysement. Leurs hiérarchies imaginées sont évidentes, car ils cherchent à découvrir d’autres mondes à travers les langues les plus prestigieuses uniquement. À aucun moment ces participants n'ont signalé leur conscience d'appartenir à une société pluriculturelle : il suffirait de se déplacer à l'intérieur du pays pour trouver d'autres langues et découvrir d'autres mondes. 


\subsection{Hiérarchies imaginées de langues}

L'apprentissage de certaines langues est considéré comme une montée dans la hiérarchie sociale. Les langues deviennent des échelons vers le haut ou vers le bas : «Apprendre une langue prestigieuse socialement est la clé de l'ascension » (Torres-Castillo, 2018).

Dans le cas du Mexique, le statut formel et informel de l'anglais est plus favorable. L'anglais s'affiche dans les cours obligatoires de plusieurs établissements scolaires. L'anglais est présenté au Mexique « comme plus prestigieux », comme langue qui assure « la fierté d'être dans le vent de la mondialisation » (Montenay, 2005 : 67). Selon Hernandez (2005), on ne peut pas discuter au Mexique de la situation du français langue étrangère (FLE) sans mentionner la présence de l'anglais, langue aussi enseignée partout dans le pays.

Depuis les années 2000, l'offre de formation en français langue étrangère dans des institutions publiques et privées s'est davantage développée. Le français fait actuellement partie des curriculums dans certains lycées comme dans l'enseignement universitaire. Depuis plusieurs années, la balance est en faveur de l'anglais et de son enseignement en tant que langue étrangère. L'ambassade de France au Mexique estime à 250000 le nombre d'étudiants du français (2013) sur le territoire mexicain.

Les enseignants ont manifesté leur intérêt pour le français car ils pensent cette langue comme un moyen de distinction parmi les Mexicains.

La participante Lorena déclare : "Parler français est avoir du prestige et appartenir à une élite». Exemple 11. (Torres Castillo, 2018 : 292)

Jaime répond «On sait, même si cela parait arrogant, le français n'est pas parlé par tous, c'est une raison élitiste et égocentrique, ma maitrise du français a été très importante et il m'a distingué parmi mes collègues et les autres enseignants de français ». Exemple 12. (Torres Castillo, 2018: 292)

Quand on leur a demandé pourquoi ils ont choisi d'enseigner le français et pas leur langue première, les réponses ont été variables.

« Je ne m'étais jamais posé cette question, pourquoi j'enseigne le français et pas l'espagnol; peut-être est-ce à cause d'un intérêt inné, qui, à travers les années et à l'aide de la licence en français, est devenu ma vocation. L'espagnol me semble aussi une belle langue et encore plus compliquée (...) On est beaucoup à parler l'espagnol, mais peu sont ceux qui ont le courage de parler une autre langue et surtout une langue qu'on n'a pas apprise depuis l'enfance ». Exemple 17 : Extrait de la réponse de Sandra à la question 2 du questionnaire de Xalapa (traduction personnelle). (Torres Castillo, 2018 : 297) 
Le français est mis en valeur, les imaginaires collectifs sont plus valorisants pour la langue française. Elle confirme que plusieurs déjà parlent espagnol.

Reyna répond: " Je n'enseigne pas l'espagnol parce que je ne me suis pas formée dans cette langue, et quand j’ai décidé de choisir ma formation, je n’appréciais pas beaucoup ma langue, mais à ce moment, si je pouvais enseigner aussi l'espagnol, je le ferais et je pense que je le ferais avec le même plaisir que celui avec lequel j'enseigne le français » Exemple 15b. (Torres Castillo, 2018 : 295)

Les remarques par rapport à sa langue première laissent voir qu'elle considère nécessaire de suivre une formation spéciale dédiée à l'enseignement de l'espagnol pour ensuite devenir enseignante. Elle ne considère pas avoir la légitimité en tant que « native » pour l'enseigner.

En ce qui concerne les langues indigènes au Mexique, il est devenu depuis 2003 le droit de tout Mexicain de s'exprimer dans sa langue première, même si celle-ci est différente de l'espagnol. Dans l'article 3 de la Loi des droits linguistiques des communautés indigènes (Ley de Derechos Lingüísticos de los Pueblos Indígenas), l'État mexicain reconnait les langues comme partie du patrimoine culturel et linguistique national. La pluralité de langues indigènes est ainsi établie comme une des principales expressions de la composition pluriculturelle de la nation mexicaine. Puis, dans l'article 4, les langues indigènes sont reconnues dans cette loi en tant que langues nationales grâce à leur origine historique, les langues indigènes ont alors la même validité dans le territoire mexicain. Dans l'article 9, on exprime le droit de tout Mexicain à communiquer dans sa langue sans aucune restriction dans des contextes publics, privés, ou à l'écrit ou oral, et dans toutes ses activités sociales, économiques, politiques ou culturelles (Gouvernement du Mexique, 2003). Malheureusement, comme nous l'avons exposé auparavant, cette belle réalité n'existe que dans le statut formel des langues indigènes. Dans le statut informel, c'est-à-dire le discours des Mexicains, on est loin de considérer les langues indigènes comme moyen d'expression dans la société mexicaine.

\section{Conclusions}

Je me suis aperçue que les images des apprenants sur le Français et sur les autres locuteurs « étrangers » relevaient d'imaginaires nourris d'une sorte d'admiration pour l'étranger. La langue espagnole et les langues indigènes étaient, à l'évidence, moins importantes à leurs yeux. De plus, l'étranger était reconnu comme « autre », mais le plus souvent comme un autre meilleur (Torres-Castillo, 2018). Les images-histoire présentées sont des éléments pour comprendre la réalité passée et actuelle mexicaine. Les locuteurs de langues indigènes sont parfois méprisés ; pas uniquement par les autres, car cette dévalorisation correspond plutôt à une autoévaluation négative. Les Mexicains préfèrent choisir les langues étrangères prestigieuses car elles sont plus valorisées dans la société mexicaine. Cependant, la vulnérabilité linguistique ne se présente pas uniquement pour les locuteurs de langues indigènes. La langue étrangère peut être à double tranchant, c'est-à-dire servir à se mettre en 
valeur et se sentir appartenir à une élite lorsqu'on se trouve entre Mexicains ; ou bien servir comme moyen pour s'auto-dévaloriser devant l'autre-étranger. 


\section{Références}

Amossy, Ruth et Pierrot Herschberg (1997), Stéréotypes et clichés, Paris, Nathan.

Barriga, Rebeca et Pedro Martín Butragueño (2015), Historia sociolingüística de México : México Prehispanico y colonial, Mexico, El Colegio de México.

Bartra, Roger (2013), «Los hijos de la Malinche », dans Margo Glantz (dir.), La Malinche sus padres y sus hijos, México, Taurus, p. 161-164.

Brotherston, Gordon (2013), « La Malitzin de los códices », dans Margo Glantz (dir.), La Malinche sus padres y sus hijos, México, Taurus, p. 7-18.

Bueno-Bravo, Isabel (2010), «El lienzo de tlaxcala y su lenguaje interno », Anales del Museo de América, $n^{\circ} 18$, p. $56-77$.

Castellotti, Véronique (2006), «Accents francophones et appropriation du français : quelles représentations? », dans Actes du colloque Français fondamental, corpus oraux, contenus d'enseignement, Lyon, ENS Éditions, p. 8-10.

Castellotti, Véronique (2011), « Natif, non natif ou plurilingue : dénativiser l'enseignement des langues? », dans Fred Dervin et Vasumathi Badrinathan (dir.), L'enseignant non natif: identités et légitimité dans l'enseignement-apprentissage des langues étrangère, Bruxelles, Éditions modulaires européennes, p. 29-50.

Charaudeau, Patrick (1995), Regards croisés : perceptions interculturelles France-Mexique, Paris, Didier.

Chavez, Ezequiel (2005), "La sensibilidad del Mexicano », dans Roger Bartra (dir.), Anatomia del Mexicano, México, Plaza Janéz, p. 22-45.

Dabène, Louise (1997), « L'image des langues et leur apprentissage », dans Marinette Matthey (dir.), Les langues et leur image, Neuchâtel, IRDP, p. 19-24.

De la Torre, Juan (1893), El amigo de las niñas mexicanas : libro de lectura corriente especial para las escuelas de las niñas, México, Libreria Nueva.

Galisson, Robert et Daniel Coste (1976), Dictionnaire de didactique des langues, Paris, Hachette.

Garro, Elena (2003), La maitresse d'Ixtepec, Paris, L'Herne.

Goi, Cécile et Torres-Castillo, Claudia (2013), « Réflexivité altéritaire et rencontre interculturelle dans un projet de recherche international », dans Véronique Castellotti (dir.), Le français dans la mondialisation, Bruxelles, Éditions modulaires européennes, p. 379-404.

Gonzalbo, Pilar (2012), "Afectos e intereses en el matrimonio en la ciudad de México », Aleph Ciencias Sociales Colégio de México, disponible sur http://aleph.academica.mx/jspui/bitstre am/56789/29225/1/56-224-2007-1117.pdf. [Page consultée en mai 2020.] 
Gouvernement du Mexique (2003), Ley de Derechos Lingüísticos de los Pueblos Indígenas, disponible sur www.gob.mx/cms/uploads/attachment/file/30119/DerechosLinguisticos.pdf. [Page consultée le 11 novembre 2020.]

Gruzinski, Serge (1990), La guerre des images, Paris, Fayard.

Gschwind-Holtzer, Giséle (1981), Analyse sociolinguistique de la communication et didactique, Paris, Didier.

Gülich, Elisabeth (1997), « Les stéréotypes nationaux, ethniques et culturels : une recherche pluridisciplinaire », dans Marinette Matthey (dir.), Les langues et leurs images, Neuchâtel, IRDP, p. 35-57.

Hernández, Magdalena (2005), La politique linguistique et l'avenir du français au Mexique : étude du cas de l'Université de Veracruz, thèse de doctorat, Birmingham, Aston University.

Johansson, Patrick (2004), « Diégesis iconográfica : la gestación semiológica del fuego uráneo en el texto pictórico del Códice Boturini », dans Patrick Lesbre et Marie-José Vabre (dir.), Le Mexique préhispanique et colonial, Paris, L'Harmattan, p. 189-204.

Johansson, Patrick (2014), « Lenguas y literaturas indígenas mexicanas », dans Diego Valades (dir.), Lengua oficial y lenguas nacionales en México, México, AML, p. 50-99.

Montenay, Yves (2005), La langue française, Paris, Les Belles Lettres.

Ramírez, Santiago (2005), "Psicoanálisis del mestizaje », dans Roger Bartra (dir.), Anatomia del Mexicano, México, Del Bolsillo, p. 237-242.

Riba, Patrick (2011), «L'enseignement du français langue étrangère au Mexique : un regard à la lumière des statistiques du DELF et du DALF, certifications officielles du ministère français de l'Éducation nationale », Synergies Mexique, nº 1, p. 47-53.

Saenger, Cony et Yurén, Teresa (2006), "La Mirada del Otro en los Dispositivos de Formación de Lenguas Extranjeras: Isomorfismos de la Política Lingüística y la Mediación », Education Policy Analysis Archives/Archivos Analíticos de Políticas Educativas, vol. 14, n²5, disponible sur www.redalyc.org/articulo.oa?id=275020543025. [Page consultée le 8 avril 2021.]

Sahagun, Fray Bernardino de (1880), Histoire Générale des choses de la Nouvelle Espagne, Paris, G. Masson. [Titre original : Historia general de las cosas de nueva España, 1575-1577. Traduit de l'espagnol.]

Semprini, Andrea (1997), Le multiculturalisme, Paris, Presses universitaires de France.

Thomas, Hugh et Guillaume Villeneuve (2011), La conquête du Mexique, Paris, Robert Laffont.

Torres-Castillo, Claudia (2015), «Enseignants mexicains de FLE "non-natifs": esquisse d'un métier entrecroisé, entremêlé, entrechoqué de plusieurs "cultures linguistiques" ? », dans Emmanuelle Huver et David Bel (dir.), Prendre la diversité au sérieux en didactique/didactologie des langues. Altériser, instabiliser: quels enjeux pour la recherche et l'intervention?, Paris, L'Harmattan, p. 195212. 
Torres-Castillo, Claudia (2017), Las culturas lingüísticas habitus en un mundo plurilingüe, thèse de doctorat, México, Universidad Autónoma del Estado de México.

Torres-Castillo, Claudia (2018), Enseignement du français, altérités et contacts de langues: imaginaires de professeurs mexicains, thèse de doctorat, Tours, France, Université de Tours.

Uranga, Emilio (2005), « Ontología del mexicano », dans Roger Bartra (dir.), Anatomia del Mexicano, México, Plaza Janéz, p. 145-158.

Villoro, Luis (2005), «El yo indígena », dans Robert Bartra (dir.), Anatomia del Mexicano, México, Random House, p. 203-213.

Wunenburger, Jean-Jacques (2016), L'imaginaire, 3e éd., Paris, Presses universitaires de France. [1'éd., 2003].

Zimmerman, Klaus (2004), "El Contacto de las Lenguas Amerindias con el español en México », Revista internacional de Lingüística Iberoamericana, vol. 2, n 4 (El español en contacto con otras lenguas), p.19-39.

Zimmerman, Klaus (2015), «Diglosia y otros usos diferenciados de las lenguas y variedades en el México del siglo XX: entre el desplazamiento y la revitalización de las lenguas indomexicanas », dans Historia sociolingüística de México. Vol. 2 : México contemporaneo, México, El Colegio de México.

Zuñiga, Rosa María (2003), Malinche esa ausente siempre presente, Madrid, Plaza y Valdés.

Zunzunegui, Juan-Miguel (2013), Los mitos que nos dieron trauma. México en el diván : cinco sesiones para superar el pasado, México, Grijalbo. 